how wheat plants respond to being fertilized with two different forms of nitrogen, nitrate and ammonium, under varying concentrations of atmospheric carbon dioxide.

The plants receiving ammonium responded much more to the increased carbon dioxide than did the plants receiving nitrate, the scientists found. Elevated carbon dioxide levels inhibited the processing of nitrate in the wheat leaves. When atmospheric carbon dioxide rose to nearly twice the normal level, the leaves of plants receiving ammonium increased in size by nearly $49 \%$, while plants receiving nitrate increased by only $24 \%$.

Additionally, the protein content of the wheat plants receiving ammonium increased $73 \%$ under elevated carbon dioxide, compared to only $32 \%$ for those receiving nitrate, suggesting that rising atmospheric carbon dioxide levels might diminish the nutritional quality of grain receiving nitrate fertilizer. The work has since been repeated in tomatoes, with similar results.

"We expect that the data will have real-world implications for crop production," Bloom says. "In well-drained soils generally devoted to wheat production, nitrate is the common form of nitrogen available. The study suggests that a shift to increase ammonium availability might be needed."

Furthermore, plant and tree species in natural ecosystems that depend on nitrate conversion into amino acids in their leaves are likely to be at a competitive disadvantage with those species that are either able to convert nitrate into amino acids in their roots or use ammonium as their predominant nitrogen source. "This may result in significant changes in the distribution of plants in the wild as atmospheric carbon dioxide levels continue to rise," Bloom says.

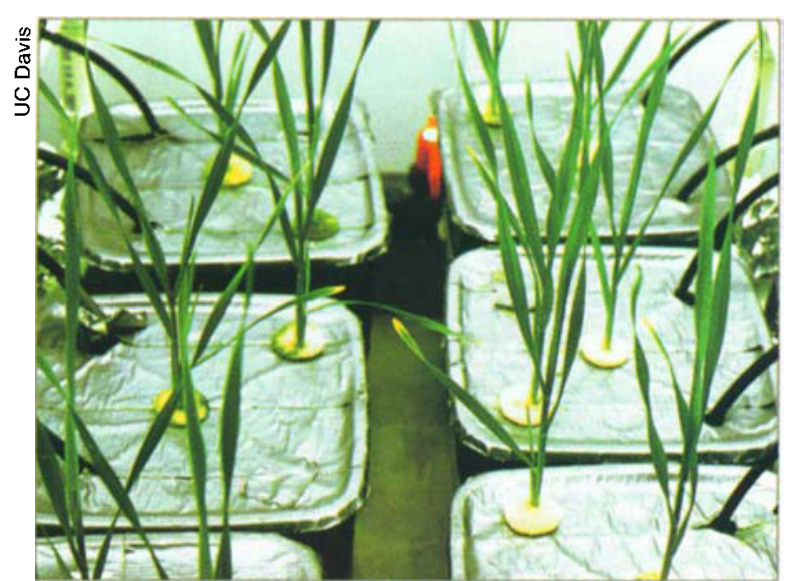

Wheat was grown in a controlled environmental chamber under elevated carbon dioxide. Plants received ammonium (left) or nitrate (right) as their sole nitrogen source. Leaves of plants receiving nitrate are yellowish-green, signaling that they are not assimilating the nutrient as well as plants receiving ammonium.

\section{Super-sensitive nitrogen dioxide detector identifies greenhouse gases}

With the aid of the most sensitive nitrogen dioxide detector in the world, UC Berkeley chemists can now quantify certain important contributors to air pollution and global warming.

Human-made nitrogen oxide compounds are emitted by automobiles, industrial smokestacks and other sources; they react with natural hydrocarbons from vegetation to produce ozone smog. "Ozone in the troposphere has doubled in the past century, contributing $10 \%$ to $15 \%$ of the human additions to the greenhouse effect," says UC Berkeley chemistry professor Ronald C. Cohen. "All of this is driven by nitrogen oxides."

Until now, however, as much as half the resulting nitrogen oxides have been unaccounted for in the atmosphere,

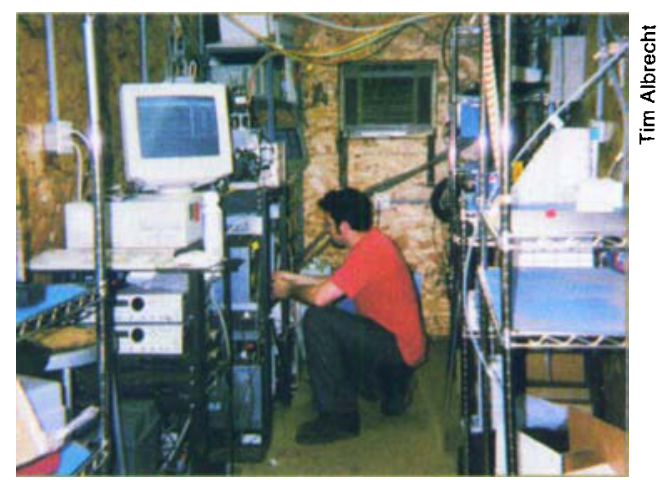

Doug Day checks the performance of the world's most sensitive nitrogen dioxide detector, in a trailer at the UC Blodgett Forest Research Center in EI Dorado County. The detector found high levels of previously unquantified organic nitrogen compounds, an observation that current models cannot adequately explain. leaving air pollution models incomplete. Today's smog monitors measure essentially the sum total of all nitrogen oxides in the air, and are unable to break them down into the specific amount of each nitrogen oxide-containing chemical.

Over about 4 years, Cohen and his laboratory colleagues developed a new detector, called thermal dissociation-laser induced fluorescence (TD-LIF), which can monitor nitrogen oxide compounds continuously with sensitivity down to 30 parts per trillion. The technique is a thousand times more sensitive than needed for today's pollution monitoring and sensitive enough for studies in remote locations.

Deploying the detector in downtown Houston and in a remote Sierra Nevada forest, the scientists detected large amounts of organic nitrogen oxide compounds called alkyl nitrates, which were thought to be only a minor constituent of smog.

"If we want to understand quantitatively the effect of local pollution on the global scale, we need to know how and in what form nitrogen oxide is transferred to the rest of the globe," Cohen says.

The research was published in the March 2002 issue of the Journal of Geophysical ResearchAtmospheres, and is supported by the National Aeronautics and Space Administration.

\section{- Compiled from UC and other news sources}

